anaesthetic gases had entered the stomach and caused this accident. Experiments have shown, however, that halothane of less than $7 \%$ concentration in $70 \%$ nitrous oxide with $30 \%$ oxygen cannot be ignited by a power source of $80-120 \mathrm{~J}$, which is much greater than that used in surgical diathermy $(10 \mathrm{~J}) .^{3}$ The presence of other gases, such as hydrogen and methane, would make an explosion possible. We postulate that fermentation can occur in an obstructed stomach and produce explosive mixtures.

We report this case to make surgeons aware that explosive gases may accumulate in the stomach when the gastric outlet is obstructed. Before operation we were unaware of the extent of the obstruction in this patient. We recommend that an obstructed stomach be completely emptied through a wide bore nasogastric tube either before or during surgery before cutting diathermy is used for gastrotomy. Alternatively, cutting diathermy could be avoided when the gastric outlet is obstructed.

We thank Mr J L Wilkins for permission to describe this case.

1 Bigard MA, Gaucher P, Lassalle C. Fatal colonic explosion during colonoscopic polypectomy. Gastroenterology 1979;77:1307-10.

2 Carroll KJ. Unusual explosion during electrosurgery. Br Med f 1964;ii:1178. 3 Churchill-Davidson HC. Flammable anaesthetic vapours. In: Wylie WD, Churchill-Davidson $\mathrm{HC}$ eds. A practice of anaesthesia. 5th ed. London: Lloyd-Luke, 1984:240-4.

(Accepted 27 October 1988)

\section{Ethnic differences in birth statistics from central Birmingham}

\section{R Griffiths, M White, M Stonehouse}

Department of Community Medicine, Central Birmingham Health Authority, Birmingham B15 2TZ

Rod Griffiths, FFCM, director of health care policy

Martin White, MB, senior house officer

Mary Stonehouse, $\mathrm{MB}$, senior house officer

Correspondence to: Dr Griffiths.

Br.Med $\mathcal{f} 1989 ; 298: 94-5$ these findings.

\section{Methods and results}

During preparation of a health profile for Central Birmingham Health Authority ${ }^{1}$ a significantly raised postneonatal mortality was found among AfroCaribbean babies compared with European babies. A higher stillbirth rate and perinatal mortality among Asian babies and a lower stillbirth rate and perinatal mortality among Afro-Caribbean babies were also noted, as has been reported by nthers. ${ }^{2}$ We report

Our analysis was based on information collected for the Birmingham births register of the university department of social medicine. This is one of few sources of data on births in the United Kingdom where the ethnic origin of both parents is recorded. The sample comprised all births to parents resident in Central Birmingham Health Authority during 1982-4 inclusive (total number 9880). This was the largest sample obtainable: data from before 1982 were not readily available owing to changes in local authority boundaries, and data from 1985 onwards were incomplete at the time of the study. For each birth the outcome at one year was classified as stillbirth; death in the first, second, third, or fourth week; or death in the remainder of the year. Each birth was classified according to the ethnic group of both parents as European (white), Asian (Indian, Pakistani, or Bangladeshi), Afro-Caribbean, oriental, or mixed (when the parents were of different ethnic origin). The last two categories were combined to form "others" as the numbers were small. Statistical analysis was by the $\chi^{2}$ test with Yates's correction. Results for births among Asians and Afro-Caribbeans were independently compared with those among Europeans.

The table shows the results. The most notable finding was a significantly higher postneonatal mortality among Afro-Caribbean babies $\left(\chi^{2}=8.95\right.$, $\mathrm{p}<0.01)$. Causes of death in the postneonatal period were identified as far as possible by examining death certificates. Twenty seven babies (out of 56) were registered as having died of the sudden infant death syndrome. Congenital abnormalities accounted for 13 deaths, respiratory and other infections for five, accidental deaths for three, and other causes for three. Five files could not be located.
Number of births in Central Birmingham Health Authority in 1982-4 by outcome and ethnic group. Figures in parentheses are mortality/ 1000 births

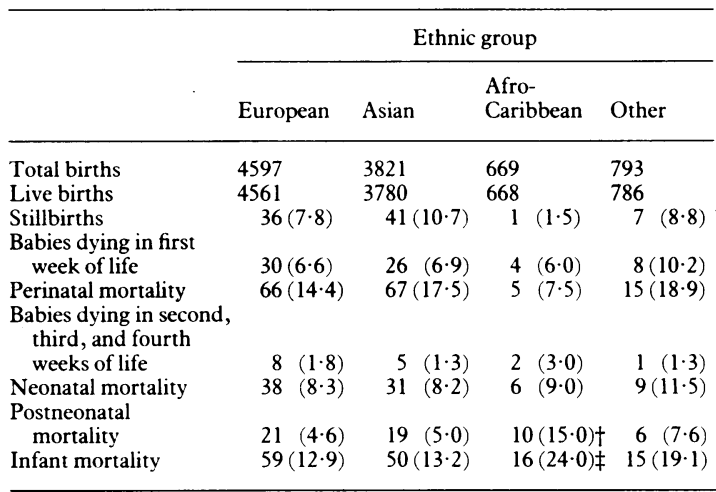

* Mortality expressed per 1000 live births except for stillbirth and perinatal mortality rates, which are expressed per 1000 total births. mortality rates, which are expr $\neq \chi^{2}=8.95,0.001<\mathrm{p}<0.01$.
$\neq \chi^{2}=4 \cdot 71,0.02<\mathrm{p}<0.05$.

\section{Comment}

Increased postneonatal mortality and an increased incidence of the sudden infant death syndrome in AfroCaribbean babies have been reported in $\mathrm{America}^{4}$ but not in Britain. Data on ethnic origin are not routinely collected when births are registered in Britain, and it is therefore unlikely that such a difference would be detected unless looked for specifically. Other districts that we contacted could not give any indication of this trend. Reasons suggested for an increased postneonatal mortality among Afro-Caribbeans include a different socioeconomic distribution from that among other ethnic groups and a lower mean birth weight. ${ }^{4}$ In our sample 387 (58\%) Afro-Caribbean babies and 2363 $(62 \%)$ Asian babies were born to parents in social class IV, V, or unclassified compared with $1444(31 \%)$ European babies. Afro-Caribbean babies also had a different birthweight distribution, with $24(3 \cdot 6 \%)$ babies weighing less than $1500 \mathrm{~g}$ compared with 38 (1\%) Asian babies and $58(1 \cdot 3 \%)$ European babies. This high incidence of very low birth weight babies among Afro-Caribbeans agrees with the findings of another study in Birmingham. ${ }^{3}$

The low perinatal mortality among Afro-Caribbeans in our study is largely attributable to the low stillbirth rate, although this value was based on small numbers and was not significant. Other studies from Britain ${ }^{3}$ and America, ${ }^{5}$ however, have shown Afro-Caribbeans to have low stillbirth rates and neonatal mortality specific to birth weight. We report this high postneonatal mortality in Afro-Caribbeans in the hope that others will review their birth statistics to see whether ethnic differences in postneonatal mortality exist. 
Further research is clearly needed to investigate this inequality and provide aetiological clues.

1 Griffiths RK, Kyle DR, Cooper RF, et al. A picture of health? Annual report 1987. Birmingham: Central Birmingham Health Authority, Department of Community Medicine, 1987.

2 Gillies DRN, Lealman TL, Lumb KM, Congdon P. Analysis of ethnic influences on stillbirths and infant mortality in Bradford 1975-1981. J Epidemiol Community Health 1984;38:214-7.
Terry PB, Condie RG, Bissenden JG, Kerridge DF. Ethnic differences in incidence of very low birthweight and neonatal deaths among normally formed infants. Arch Dis Child 1987;62:709-11.

4 Black L, David G, Brouillette RT, Hunt CE. Effects of birthweight and ethnicity on incidence of sudden infant death syndrome. $f$ Pediatr 1986;108: 209-10.

5 Hogue CJ, Buehler JW, Strauss LT, Smith JC. Overview of the national infant mortality surveillance (NIMS) project. Public Health Rep 1987;102:126-37.

(Accepted 8 June 1988; proof returned 15 December)

\section{Autoimmunity: a risk factor for allosensitisation to blood products?}

\author{
A S Wierzbicki, D M Cousins
}

\section{Department of}

Haematology, National

Hospitals for Nervous

Diseases, Queen Square,

London WC1N 3BG

A S Wierzbicki, BM, senior house officer

D M Cousins; BSC, senior

medical laboratory scientific officer

\section{Correspondence and} requests for reprints to: $\mathrm{Dr}$ Wierzbicki.

Br.Med f 1989;298:95-6 over 10 months.

\section{Case histories}

Transfusion of blood products sensitises some patients to isoantigens. We report three cases of the formation of multiple persistent isoantibodies that occurred in association with a complex of autoimmune diseases

Case 1-A 50 year old woman with a history of rheumatoid arthritis treated with various agents including prednisolone was admitted for odontoid peg surgery. She had had two pregnancies, and six years before admission she had received 5 units of packed erythrocytes during three orthopaedic operations. Clinically she had vitiligo and hypothyroidism. Initially only anti-rhesus E and anti-Duffy antibodies were detected. Postoperative transfusion was followed by a delayed haemolytic transfusion reaction at 10 days. Further surgery for a cerebrospinal fluid leak required repeat transfusion, which disclosed additional antiKell, anti-rhesus C, anti-Kidd, and HLA antibodies to $80 \%$ of a lymphocyte panel (North West Thames Regional Blood. Transfusion Centre, Edgware). Further episodes of haemolysis occurred until fully crossmatched blood (also anti-S negative) was used. She was managed symptomatically and made a good recovery.

Case 2-A 65 year old nullipara presented with a right frontal glioma and a history of aplastic anaemia managed by splenectomy (eight years). She had been treated with prednisolone five years previously. Her total transfusion history was 8 units of packed cells up to six years previously and 12 units of platelets up to nine years previously. Her autoimmunity was manifested by vitiligo, hypothyroidism, and pernicious anaemia. On admission she was anaemic $(105 \mathrm{~g} / \mathrm{l})$ and thrombocytopenic $\left(26 \times 10^{9} / 1\right)$ and was crossmatched for red cell transfusion. Repeat crossmatching two hours after platelet transfusion, when she remained thrombocytopenic, disclosed HLA antibodies. This necessitated tissue typing before revision of her bone flap. During the transfusions she had a mild trans- fusion reaction on four occasions. Eventually she recovered.

Case 3-A 51 year old woman was admitted for thymectomy after presenting with myasthenia gravis. She had a previous history of hypothyroidism and breast adenocarcinoma and was receiving long term prednisolone. She had borne three children. Her total transfusion history was 4 units of packed cells two years previously. Her thymectomy was uneventful and she did not require transfusion. She made a good recovery.

The table gives details of the antibody profiles in the three cases.

\section{Comment}

Blood product isoantibodies and autoantibodies are becoming commoner with the frequency of multiple transfusions and bone marrow transplant operations. The prevalence of isoantibodies varies from $0.5 \%$ to $3.8 \%^{1}$ or a reaction occurs to roughly one in 4000 units of blood transfused. ${ }^{2}$ Usually, however, antibodies are to only one blood group-for example, rhesus-and they occur in $10-36 \%$ of multitransfused patients. ${ }^{34}$ Rarely two blood groups are affected $(10 \%$ of delayed transfusion reactions), but three or more are very rare (1\%). Most of these antibodies decline in titre over four years to $60-80 \%$ of their original prevalence. ${ }^{5}$ AntiKidd and anti-rhesus $\mathrm{E}$ are commonly seen in allosensitisation and also reappear on restimulation, as occasionally does anti-Duffy. ${ }^{4}$

Our patients were unusual in that their exposure to blood products was small and had occurred several years previously. Multitransfusion studies cite transfusions of 10-20 units one year previously. ${ }^{3}$ The three patients were immunosuppressed with steroids, which should have reduced their predisposition to allosensitisation.

All three patients were of the sex and age groups particularly affected by autoimmune diseases, and all showed pronounced immune dysfunction manifested by formation of multiple autoantibodies. We suggest that this defect in their immune suppressor mechanisms might also have predisposed them to the induction and persistence of antibodies against blood products. This type of patient might therefore benefit from more accurate crossmatching for minor blood groups to avoid the possibility of later delayed haemolytic transfusion reactions.

ADDENDUM - Since submitting this report we have seen a further case of a 59 year old nullipara with

Results of blood crossmatching and screening for autoantibodies and isoantibodies in three patients

\begin{tabular}{|c|c|c|c|}
\hline & Case 1 & Case 2 & Case 3 \\
\hline Erythrocyte crossmatch & Normal & Normal & Normal \\
\hline Direct antiglobulin test result & Negative & Negative & Negative \\
\hline Autoantibodies (titre) & $\begin{array}{l}\text { Thyroglobulin }(1 / 640) \text {; thyroid } \\
\text { microsome }(1 / 320) \text {; antinuclear } \\
(1 / 80) \text {; rheumatoid antinuclear } \\
(1 / 64)\end{array}$ & $\begin{array}{l}\text { Thyroglobulin }(1 / 320) \text {; thyroid } \\
\text { microsome }(1 / 160) \text {; intrinsic } \\
\text { factor; parietal cell }\end{array}$ & $\begin{array}{l}\text { Thyroglobulin (1/640); thyroid } \\
\text { microsome (1/640); antinuclear } \\
\text { (1/320); striated muscle; } \\
\text { acetylcholine receptor }\end{array}$ \\
\hline Isoantibodies & $\begin{array}{l}\text { Rhesus C, E; Duffy; Kell; Kidd; } \\
\text { HLA }(80 \%)\end{array}$ & HLA $(90 \%)$ & Rhesus C, D; Kidd \\
\hline
\end{tabular}

